\title{
Robotic radical thymothymectomy for thymomatous myasthenia gravis in the elderly: case report and mini-review
}

\author{
François Montagne ${ }^{1}$, David Delacour ${ }^{2}$, Marion Fommarty $^{1}$, Zied Chaari ${ }^{1,2,3}$, Eloise Papet ${ }^{1}$, Jean-Marc Baste ${ }^{1,4} \wedge$ \\ ${ }^{1}$ Department of General Thoracic Surgery, Rouen University Hospital, Rouen, France; ${ }^{2}$ Department of Radiology, Cardiac Imaging Unit, University \\ Hospital of Rouen, Rouen, France; ${ }^{3}$ Department of Thoracic and Cardiovascular Surgery, Habib Bourguiba University Hospital, Sfax, Tunisia; \\ ${ }^{4}$ INSERM U1096, Haute Normandie Research and Biomedical Innovation, Normandie University, Rouen, France \\ Contributions: (I) Conception and design: F Montagne, JM Baste; (II) Administrative support: F Montagne, D Delacour, JM Baste; (III) Provision of \\ study materials or patients: F Montagne, D Delacour, JM Baste; (IV) Collection and assembly of data: F Montagne, JM Baste; (V) Data analysis and \\ interpretation: F Montagne, JM Baste; (VI) Manuscript writing: All authors; (VII) Final approval of manuscript: All authors. \\ Correspondence to: Jean-Marc Baste, MD, PhD. Department of General Thoracic Surgery, Rouen University Hospital, 1 rue de Germont, 76000 \\ Rouen, France. Email: jean-marc.baste@chu-rouen.fr.
}

\begin{abstract}
Thymectomy remains a therapeutic challenge in elderly patients with thymoma associated with myasthenia gravis (MG). Thymectomy, in the multimodality therapy of these patients should be discussed by multidisciplinary expert teams. Available data show that robotic thymectomy seems to be as safe and as effective as an open procedure with better short-term outcomes and with at least the same quality of oncologic resection. Nevertheless, data are limited on the safety and effectiveness of robotic thymectomy in elderly patients. We present here, our surgical technique of robotic radical en bloc thymothymectomy that we use in all our patients with thymoma associated with MG, with particularities related to elderly patients. We also review the medical and surgical literature on robotic thymectomy for thymoma and MG. The robotic platform allows us to "mimic an open procedure by a minimally invasive approach", and thus, we are able to perform a complete and radical thymothymectomy comparable to open procedure with the same quality of oncologic resection, but with the advantages of a minimally invasive approach regarding short-term outcomes.
\end{abstract}

Keywords: Minimally invasive surgery; robotic thymectomy; myasthenia gravis (MG); thymomatous myasthenia gravis; case report

Received: 11 May 2020; Accepted: 24 June 2020; Published: 20 April 2021.

doi: $10.21037 /$ jovs-20-128

View this article at: http://dx.doi.org/10.21037/jovs-20-128

\section{Introduction}

Despite technological progress and surgical advances, some procedures remain therapeutic challenges in some populations. Thymectomy in elderly patients with thymoma associated with myasthenia gravis (MG) is one such example.

Most thymomas are found in adult patients, with a peak incidence in the $7^{\text {th }}$ decade of life, but thymomas associated with $M G$ are typically diagnosed in the $5^{\text {th }}$ decade. MG is a rare autoimmune disorder characterized by muscle weakness caused by the production of autoantibodies against acetylcholine receptors or other molecular components at the neuromuscular junction. MG is the most common parathymic syndrome and occurs in $45 \%$ of patients with thymoma, more often B-type thymomas (1). MG incidence is increasing worldwide, and also in the elderly population (2).

The treatment of thymoma involves a combination

^ORCID: 0000-0002-1739-4523. 
of surgery, radiotherapy and chemotherapy, and each therapeutic combination is guided by the stage of the disease (3). En bloc thymothymectomy is the surgical procedure recommended for stage I and II thymoma and is defined as the removal of the thymoma, the thymus with the anterior mediastinal fatty tissue, and the mediastinal pleura. For stage III and IV thymoma, according to the Masaoka Koga classification (4), surgery-en bloc resection of the tumor and of the adjacent overgrown structures-is one treatment in the overall multimodality therapy.

In thymoma associated with MG, the oncologic imperative of the thymoma remains, but there is also the question of $M G$ with the existence of thymic islands located in the pericardial and perithymic fatty tissue. Thymectomy is performed as part of the multimodality therapy of MG since Blalock et al. (5) first reported a complete remission of MG after thymectomy in 1939. Thymectomy must always be adapted to the patient in order to perform the most "complete" or "radical", but also the least morbid resection, and accounting for intensive care monitoring in case of a postoperative myasthenic crisis. The question of the preservation of the phrenic nerve is one such an example, it's crucial to preserve it in cases of MG, but for advanced stages of thymoma, it could be spared, instead of being cut, at the expense of more local recurrences, but without affecting overall survival (6).

Many approaches have been described such as the "maximal" thymectomy by an en bloc transcervicaltranssternal resection of Jaretzki et al. (7) during the 80's. During the 90 's, at the beginning of minimally invasive surgery, video-assisted thoracoscopic surgery (VATS) thymectomy was developed $(8,9)$, and at the end of the 90's, Zielinski et al. (10) developed a transcervical-subxiphoidvideothoracoscopic approach for non-thymomatous MG. Yoshino et al. (11) were the first to report the successful use of the da Vinci robotic system with a thymothymectomy procedure for a stage I thymoma, and similarly for Ashton et al. (12) with a thymectomy for a non-thymomatous MG. Today, it is accepted that robotic-assisted thoracoscopic surgery (RATS) thymectomy is a safe procedure, with fewer complications and adverse events, with a lower positive margin rate for a comparable operative time compared to open thymectomy (13) for early-stage thymomas and also for larger thymomas superior to $6 \mathrm{~cm}$ wide (14). When compared to VATS, RATS allows the surgeon to mimic open thymectomy, with the benefits of both a minimally invasive approach and an open sternotomy.

There are few data concerning thymectomy for elderly MG patients. Recently Li et al. (2) reported good results in favor of performing robotic thymectomy for MG patients older than 60 years with a statistically significant steroidsparing effect. Our clinical case echoes the article of $\mathrm{Li}$ et al. (2), with a particular question, "How far to go?". In an elderly patient with $M G$, with a lesion that seemed to be a thymoma on CT-scan, how should we perform the resection of the pericardial fatty tissue? What are the limits of our resection? Should we always be "maximalist" as Jaretzki for example? We present the following case in accordance with the CARE reporting checklist (available at https://jovs. amegroups.com/article/view/10.21037/jovs-20-128/rc).

\section{Clinical case description}

An 80-year-old woman, usually treated for high blood pressure, was referred to neurology unit. She presented a left eye ptosis associated with fluid dysphagia evolving for 4 months, and her clinical condition corresponded to Myasthenia Gravis Foundation of America (MGFA) grade IIIA (15). The diagnosis of MG was confirmed by laboratory investigation-sero-positivity for the autoantibody against acetylcholine receptor-and by electromyographypost-synaptic decrement signal. The first CT-scan only showed hyperplasia of the mediastinal fatty tissue, without thymoma-like tumor. Treatment with ammonium chloride allowed rapid correction of her symptoms. Six months later, she had a hip prosthesis, and was treated for a zona of the right buttock during her postoperative rehabilitation. Back home, 2 months after her hip surgery, she had an episode of myasthenic crisis with respiratory failure that required mechanical ventilation. She was intubated for 14 days, a tracheostomy was done and finally removed one month after symptoms onset. During the myasthenic crisis, she was treated with intravenous infusion of immunoglobulin for 3 days and corticotherapy was introduced. A new CT-scan was done-2 years after her first neurologic consultationand described an anterior mediastinal tumor comparable to an early-stage thymoma, measuring $35 \mathrm{~mm}$ by $25 \mathrm{~mm}$ by $19 \mathrm{~mm}$ (Figures 1,2). Four months after the resolution of her myasthenic crisis, after multidisciplinary discussion, the patient was referred to our thoracic unit. Symptoms of MG were under control with ammonium chloride- $10 \mathrm{mg}$ 3 times a day-and a corticosteroid therapy-25 mg in the morning. We decided to perform a RATS radical thymothymectomy by a left-sided approach, with two objectives. The first one was to protect the patient from scalability of the thymoma and the second one was to stop her steroid therapy. The patient gave her informed consent 

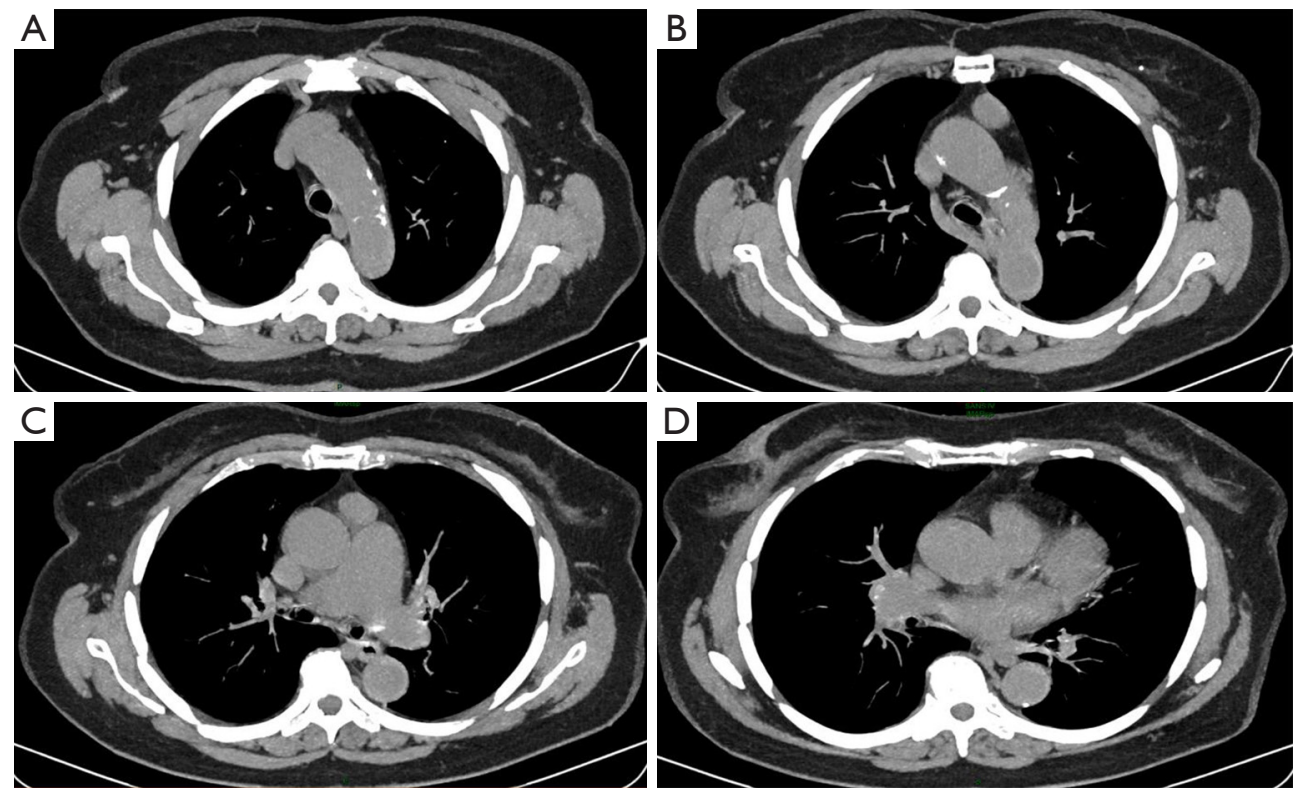

Figure 1 CT scan of our patient with transversal slides. (A) Drainage level of the left internal mammary vein in the superior vena cava; (B) upper part of the thymoma; (C) pulmonary artery trunk; (D) inferior part of the anterior mediastinal fatty tissue.

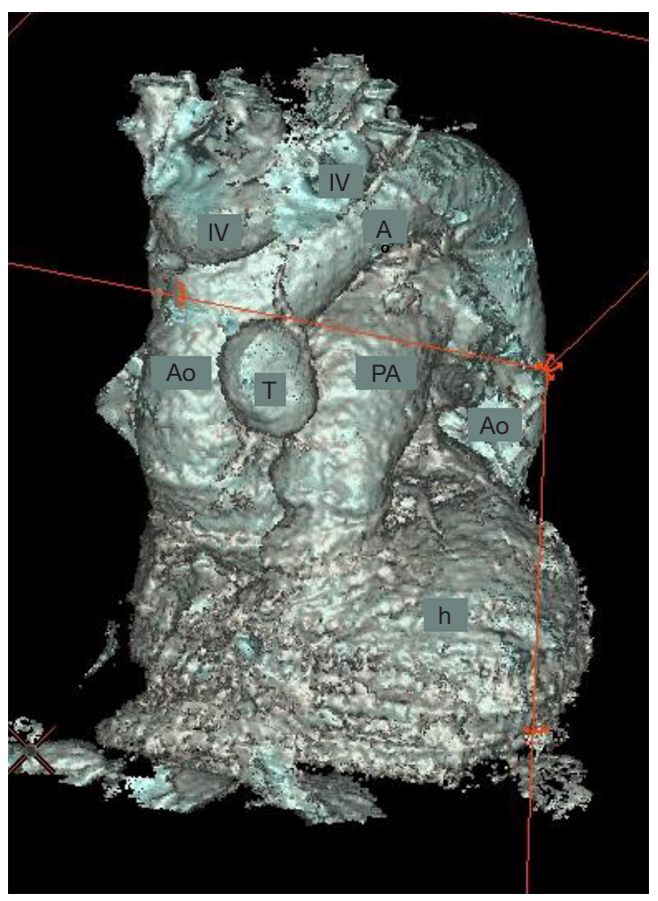

Figure 2 Three-dimensional representation of the thymoma (T) on the heart (h), and its vessels, with the pulmonary artery (PA), the aorta (Ao), and the innominate vein (IV). to use and published medical information and the surgical procedure video recording. All procedures performed in this study were in accordance with the Helsinki Declaration (as revised in 2013).

\section{Pre-operative preparation}

\section{The choice of the side}

The side approach is based on the presence of unilateral tumor predominance, the size and the shape of the thorax, the size of the heart and also the surgeon's experience. We usually perform a left-sided approach-for $80 \%$ of our patients-in order to better control the upper part of the left phrenic nerve, but, the disadvantage of the left approach is to dissect tangentially the innominate vein. Conversely, with a right-sided approach, the innominate vein may be dissected more safely but the left phrenic nerve may be injured during the dissection of the left upper horn due to its lateral situation. For this case of a left-sided thymoma, we performed a left-sided approach.

\section{Patient installation}

Under general anesthesia, the patient was ventilated through a left-sided double lumen endotracheal tube. The 


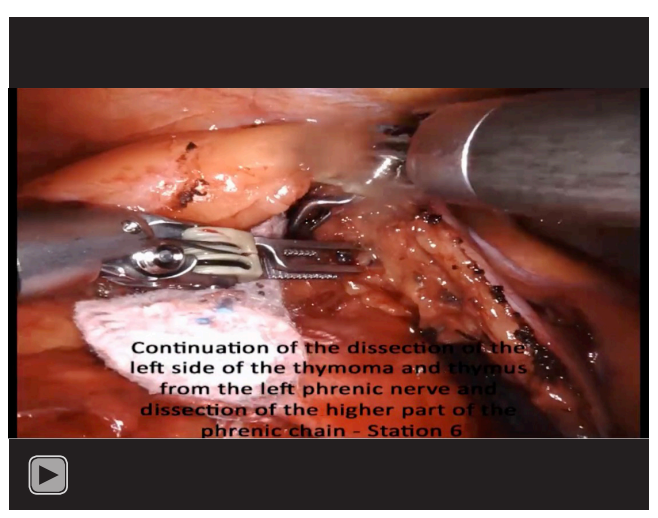

Video 1 Robotic radical Thymothymectomy for a thymomatous myasthenia gravis in an elderly patient. Surgical procedure performed with the da Vinci X platform.

patient was placed supine with the body moved to the left edge of the operating table. Her left arm was parallel to the table, but in a lower position, in order to facilitate the left-sided thoracic access, and her right arm was placed alongside her body. The left shoulder should be positioned as low as possible. One of the most common technical issues is to have insufficiency clearance between the cranial trocar and the shoulder, when this arm is directed medially. The operative body position was "fixed" using a vacuum beanbag. The skin was marked at the position of the trocar incisions and the sternotomy incision. The operative field was scrubbed, sterilized, and draped exposing the chest from the left posterior axillary line to the right anterior axillary line to ensure enough area for the 3 robotic ports and the assistant port and also to convert to sternotomy if necessary.

\section{Equipment preference and Robotic setting}

We use now the da Vinci X System (Intuitive Surgical, Sunnyvale, CA, USA). We first placed an $8 \mathrm{~mm}$ port at the fifth intercostal space on the anterior axillary line and inserted the $8 \mathrm{~mm} 30^{\circ}$ angled da Vinci camera before insufflating carbon dioxide $\left(\mathrm{CO}_{2}\right)$ to a pressure of $5 \mathrm{mmHg}$ at the beginning through this port. Under vision control, with the $30^{\circ}$ up camera, we placed the cranial port in the third intercostal space medially to the anterior axillary line, and the caudal port in the fifth intercostal space medially to the mid-clavicular line. If, there is insufficiency clearance between the cranial port and the shoulder, a long robotic port may be helpful. The $10 \mathrm{~mm}$ assistant- port, dedicated to $\mathrm{CO}_{2}$ insufflation using the iFS AirSeal ${ }^{\mathrm{TM}}$ system, was then inserted medially to the optical-trocar in the sixth intercostal space. Then, the patient-cart (Intuitive Surgical, Sunnyvale, CA, USA) was docked by aligning the thymoma, between the patient cart and the camera. Under vision control, with the $30^{\circ}$ up camera, we introduced the Maryland bipolar forceps (Intuitive Inc., Sunnyvale, CA, USA) into the cranial port and the fenestrated bipolar forceps (Intuitive Inc., Sunnyvale, CA, USA) into the caudal port for a left-sided approach. The $\mathrm{CO}_{2}$ insufflating pressure was increased to a maximum of 8 to $10 \mathrm{mmHg}$.

\section{Surgical technique and procedure}

\section{Tips and tricks, "Basics of anterior mediastinal robotic dissection"}

In our opinion, anterior mediastinal robotic dissection complies with 4 rules: "en bloc"; "no touch"; "stay and play"; "the right camera angle for each dissection area".

We usually perform an en bloc resection of the thymus, and the thymoma with its fatty tissue and also use a "notouch" thymomectomy technique to minimize tumoral seeding and bleeding. We repress the thymus by using the Endowrist system and the back of our forceps and by using a rolled compress, rather than grasping it. When the optical camera is correctly placed, we usually "stay and play", we dissect everything that can be easily dissected, before moving the camera, to reduce unnecessary and dangerous forceps movements. During each step of the dissection, it is important to use the right camera angulation. The $30^{\circ}$ down camera is used during the dissection of the thymus from the pericardium, near the phrenic nerve, or in the contralateral pleural space cavity. The $30^{\circ}$ up camera is used during the dissection of the thymus from the sternum, from the opposite mediastinal pleura, in the angle between the left phrenic nerve and the sternum and during the dissection of the upper thymic poles.

\section{Surgical technique of a radical thymectomy and thymomectomy:}

The first step is the exploration of the left thoracic pleural space, before beginning the resection. A radical thymectomy including a thymomectomy (Video 1) begins by dissecting the left-sided pericardiophrenic fatty tissue from the pericardium, the phrenic nerve, and the opposite mediastinal pleura. Before dissecting this fatty tissue from 


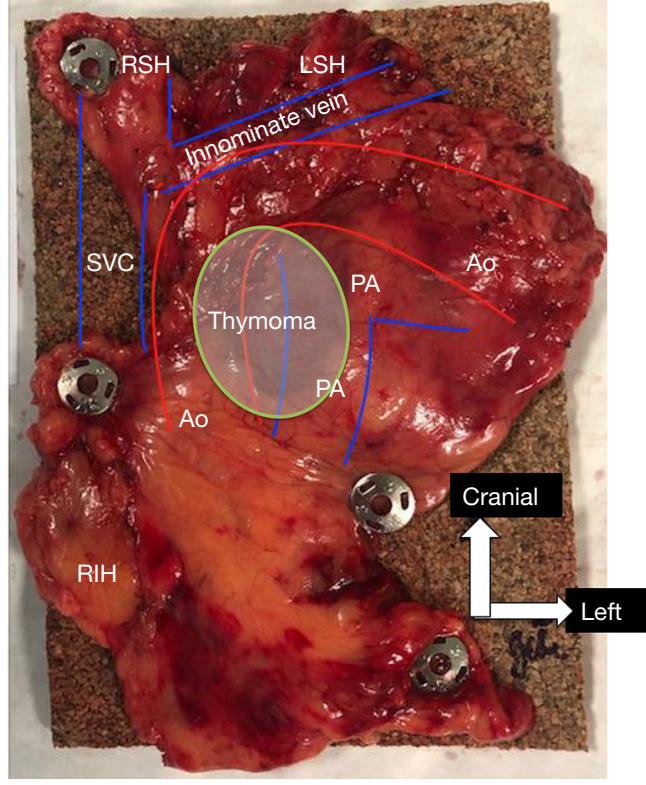

Figure 3 Specimen with anatomical landmarks: thymoma; innominate vein; aorta (Ao); pulmonary artery (PA); superior vena cava (SVC); right superior thymic horn (RSH); left superior thymic horn (LSH); right inferior thymic horn (RIH); and the pericardial anterior fatty tissue.

the sternum, it is important to completely dissect it from the pericardium as this facilitates its exposure. When the inferior side is dissected, the dissection can be continued on the contralateral mediastinal pleura and the sternum.

Going up, we continue the dissection of the thymus and the thymoma. We continue first on the pericardium side, then near the phrenic nerve and after against the contralateral mediastinal pleura and the sternum. During the dissection from the phrenic nerve, extreme caution is required to prevent any mechanical or thermal injury. We leave some fatty tissue on the nerve instead of resecting this nerve with the thymoma if the phrenic nerve is free of tumoral infiltration.

The dissection progresses cautiously in the angle between the left phrenic nerve and the sternum, and slowly, until the dissection of the innominate vein. In this area the phrenic nerve, the innominate vein, or the internal mammary veins could be injured. The dissection is done in the lower part, by taking care not to injure the thymic vein, and in its upper part by the resection of the left and the right upper horns and the fatty tissue from the thyrothymic ligament. Upper horns are dissected by gentle caudal traction and arteries and venous branches are electrocoagulated. When the upper thymic horns are completely dissected, the dissection of the innominate vein is continued in the direction of the superior vena cava by gentle caudal traction of the thymus. During this step, the thymus can be gently removed from the right pleural space. To improve the visualization of the phrenic nerve, the left angle between the phrenic nerve and the sternum, or the pericardium side, the dissected specimen can be mobilized in the right pleural space.

The dissection is continued in the angle between the innominate vein and the superior vena cava and the sternum, until the end of the dissection. In order to prevent any right-sided phrenic nerve, the $30^{\circ}$ down camera can be used at the end of the dissection of the fatty tissue from the superior vena cava.

\section{End of the robotic procedure}

The en bloc specimen of radical thymectomy including the thymoma is placed at the left apical pleural cavity. The operating field is examined to ensure that there is no bleeding, no innominate injuries or no phrenic nerve injuries. An Endobag (Applied, Rancho Santa Margarita, CA, USA) is used to extract the specimen. It is important to perform an accurate utility thoracotomy incision in order to avoid any capsule breach of the thymoma. This incision is made by removing the assistant trocar. A 20 Portex $^{\circledR}$ (Smiths Medical, Minneapolis, MN, USA) chest tube is inserted and placed transmediastinally in order to drain the two pleural cavities. Finally, the left lung is inflated under vision control. Thoracic drainage can be avoided by removing the thoracic chest tube at the last skin suture in straightforward cases if the patient is free of chronic obstructive pulmonary disease or emphysema. But for MG patients, the chest tube can be removed at postoperative day 1 because they will be monitored in ICU and in our thoracic unit. Port incisions are closed with absorbable sutures. The specimen (Figure 3) is disposed on an anatomical support.

\section{Post-operative management}

After surgery, the patient was monitored in the postoperative intensive care unit during the first 24 hours and then in our thoracic unit before she was discharged home at postoperative day 4 . The chest tube was removed at postoperative day 1 . The patient ate early in a chair at postoperative day 0 , and walked at postoperative day 1 . The patient's treatment for MG was not changed during her hospital stay. 


\section{Discussion}

An en bloc extended thymothymectomy should be performed by an experienced thoracic surgeon (16) in MG patients with a thymoma in order to completely remove the tumor. Minimally invasive procedures as VATS and RATS remain controversial for thymic malignancies (17) due to the fear of a possible breach of the tumor capsule precipitating local and pleural seeding and the uncertainty of acquiring sufficient oncologic margins.

Marulli et al. (18) in 2016 reported a multi-institutional European experience of robotic thymectomy for thymoma, including 134 RATS procedures, 52\% with MG. Twelve patients $(8.9 \%)$ were converted to open surgery for technical reasons, but there was no vascular or nerve involvement. Postoperative complication rate was $17.1 \%$. The mean diameter of the resected thymoma was $4.4 \mathrm{~cm}$, with Masaoka stage I for 46 patients (34.4\%), stage II for 71 patients $(52.9 \%)$, stage III for 11 patients $(8.3 \%)$ and $\mathrm{IVa} / \mathrm{b}$ for 6 patients (4.4\%). The 5 -year survival rate was $97 \%$ and only one patient had a pleural recurrence.

In a recent meta-analysis and systematic review including 30 studies with 2,038 patients with thymic malignancies, VATS and RATS approaches allowed safe resection with the same oncologic outcomes compared to an open procedure (17) in Masaoka stage I and II thymic malignancy. In the minimally invasive group, authors observed significantly less blood loss $(\mathrm{P}<0.001)$ and a shorter length of hospital stay $(\mathrm{P}<0.001)$ but not a significantly lower rate of postoperative pulmonary $(\mathrm{P}=0.64)$ and cardiac complications $(\mathrm{P}=0.52)$ with VATS and RATS thymectomy compared to the open group. Nevertheless, VATS and RATS allowed the same quality of oncologic resection, without differences observed in $\mathrm{R} 0$ margin resections $(\mathrm{P}=0.60)$ and locoregional recurrences $(\mathrm{P}=0.46)$ compared to open procedure. Moreover, in the subset of patients with Masaoka stage I or II thymic malignancies, there was no difference in RO margin resections $(\mathrm{P}=0.88)$ and locoregional recurrence rate $(\mathrm{P}=0.39)$. When RATS thymectomy was compared to VATS thymectomy, there was no statistical difference concerning short-term outcomes reported as blood loss, conversion rate, postoperative complications and hospital length of stay except a longer operating duration in the RATS group $(19,20)$.

RATS offers the advantages of a minimally invasive approach as VATS, but its ergonomic benefits overcome the technical and methodological limitations of VATS (20), and finally RATS allows surgeons to "mimic an open procedure by a minimally invasive approach". The robotic surgical system with its high-resolution, three-dimensional vision, and its improved dexterity of instruments with 7 degrees of freedom allowing complex three-dimensional movements, has significantly increased the safety and the oncologic effectiveness of robotic thymectomy. Moreover, for large thymomas exceeding $4 \mathrm{~cm}$, the robotic approach is safe and effective (14) compared to open procedure, with less blood loss $(\mathrm{P}=0.001)$, shorter length of hospital stay $(\mathrm{P}=0.034)$, low conversion rates, $15 \%$, without any major vascular injuries, and one of the most important criteria of oncologic quality, no difference concerning R0 margins $(\mathrm{P}=0.62)$. Rather than the absolute size of the thymoma, it is important to evaluate the ratio between the size of the thymoma and the size and the shape of the thorax. Thus, a lesion of the same size, for example $4 \mathrm{~cm}$, could be easily removed from a patient with a large chest, e.g., in a young man of $185 \mathrm{~cm}$, but represent a surgical challenge in a patient with a thinner thorax, e.g., in an elderly woman of $160 \mathrm{~cm}$ with a "big heart".

Tips and Pitfalls, and other surgical considerations of our case warrant discussion. A robotic approach was obvious for this elderly patient with early-stage thymoma associated with MG, and the real challenge was to perform the adequate resection of the thymoma, the thymus and the anterior mediastinal fatty tissue with the least possible risk. Our functional priority here was, and still is to preserve the left and right phrenic nerves. Therefore, we decided to keep a small margin of fatty tissue between the nerve and our resection in order to prevent any lesion. Our first priority is to prevent any vascular injury. This same margin should also be kept in the innominate vein and the superior vena cava if there is a high risk of vascular injury. The dissection of the cranial fatty tissue should be cautious, millimeter per millimeter to avoid any bleeding complications. During the dissection of the innominate vein, we usually electro coagulate thymic veins away from the innominate vein keeping a stump that can be compressed if necessary. Our second priority is oncology, by preventing tumor seeding by a capsule wound. Therefore, we usually perform a "no touch technique" and only manipulate fatty tissue so that the thymoma is never exposed, except on its pericardial side. Moreover, we manipulate the thymus and the fatty tissue with great caution. In elderly patients, the resection of the thymoma is first and foremost essential and there is no need to take any extra risk by dissecting the phrenic nerves, the innominate vein and the superior vena cava. At the end of the procedure when we extract the thymoma, we always use 
a bag, and we enlarge the incision of the assistant trocar in order to facilitate the extraction of the resected specimen to avoid any capsule breach that may complicate the pathology analysis.

Some medical considerations of our case also warrant discussion. A robotic approach was obvious for this elderly patient with early-stage thymoma associated with MG to facilitate recovery. At the present time, her corticosteroid therapy was interrupted without any complications. The pathology analysis revealed a B1/B2 thymoma, stage I according to the Masaoka-Koga classification and stage pT1aN0 according to the WHO classification. The nodes were free of metastasis. Many microscopic thymic cysts were observed around the thymoma.

\section{Conclusions}

Robotic radical thymothymectomy seems to be safe and effective in the multimodality therapy of thymoma associated with MG in elderly patients. These patients should be evaluated by a multidisciplinary team, and then referred to an expert surgical team. This procedure must be well prepared and performed in compliance with 4 rules: "no touch", "en bloc resection", "stay and play", and "the right camera angle for each surgical area". RATS allows us to "mimic an open procedure by a minimally invasive approach", and thus, we are able to perform a complete and radical thymothymectomy comparable to open procedure with the same quality of oncologic resection, but with the advantages of a minimally invasive approach regarding short-term outcomes.

\section{Acknowledgments}

The authors are grateful to Nikki Sabourin-Gibbs (Rouen University Hospital) for her help in editing the manuscript. Funding: None.

\section{Footnote}

Provenance and Peer Review: This article was commissioned by the the editorial office, Fournal of Visualized Surgery for the series "Robotic Assisted Thoracic Surgery: Adavanced Procedures in Lung and Mediastinum: From Postinduction TTT (immunotherapy) to Sleeve Resection, Complex Segmentectomies and Extended Thymectomy for Myasthenia Gravis". The article has undergone external peer review.
Reporting Checklist: The authors have completed the CARE reporting checklist. Available at https://jovs.amegroups. com/article/view/10.21037/jovs-20-128/rc

Conflicts of Interest: All authors have completed the ICMJE uniform disclosure form (available at https:// jovs.amegroups.com/article/view/10.21037/jovs-20-128/ coif). The series "Robotic Assisted Thoracic Surgery: Adavanced Procedures in Lung and Mediastinum: From Post-induction TTT (immunotherapy) to Sleeve Resection, Complex Segmentectomies and Extended Thymectomy for Myasthenia Gravis" was commissioned by the editorial office without any funding or sponsorship. JMB served as the unpaid Guest Editor of the series. JMB reports personal fees from Intuitive Surgery, personal fees from Medtronic, personal fees from Jonhson, outside the submitted work. The authors have no other conflicts of interest to declare.

Ethical statement: The authors are accountable for all aspects of the work in ensuring that questions related to the accuracy or integrity of any part of the work are appropriately investigated and resolved. All procedures performed in this study were in accordance with the Helsinki Declaration (as revised in 2013). The patient gave her informed consent for this publication.

Open Access Statement: This is an Open Access article distributed in accordance with the Creative Commons Attribution-NonCommercial-NoDerivs 4.0 International License (CC BY-NC-ND 4.0), which permits the noncommercial replication and distribution of the article with the strict proviso that no changes or edits are made and the original work is properly cited (including links to both the formal publication through the relevant DOI and the license). See: https://creativecommons.org/licenses/by-nc-nd/4.0/.

\section{References}

1. Detterbeck FC, Parsons AM. Thymic tumors. Ann Thorac Surg 2004;77:1860-9.

2. Li F, Takahashi R, Bauer G, et al. Results of Robotic Thymectomy Performed in Myasthenia Gravis Patients Older Than 60 Years at Onset. Ann Thorac Surg 2019;108:912-9.

3. rythmic.org. Referentiel De Prise en Charge Des Tumeurs Thymiques 2020.

4. Detterbeck FC, Nicholson AG, Kondo K, et al. The Masaoka-Koga stage classification for thymic malignancies: 
clarification and definition of terms. J Thorac Oncol 2011;6:S1710-6.

5. Blalock A, Mason MF, Morgan HJ, et al. Myasthenia Gravis and Tumors of the Thymic Region. Ann Surg 1939;110:544-61.

6. Hamdi S, Mercier O, Fadel E, et al. Is sacrifying the phrenic nerve during thymoma resection worthwhile? Eur J Cardiothorac Surg 2014;45:e151-5.

7. Jaretzki A 3rd, Wolff M. "Maximal" thymectomy for myasthenia gravis. Surgical anatomy and operative technique. J Thorac Cardiovasc Surg 1988;96:711-6.

8. Yim AP, Kay RL, Ho JK. Video-assisted thoracoscopic thymectomy for myasthenia gravis. Chest 1995;108:1440-3.

9. Rückert JC, Gelbert K, Müller JM. Operative technique for thoracoscopic thymectomy. Surg Endosc 1999;13:943-6.

10. Zielinski M, Kużdżał J, Szlubowski A, et al. Transcervicalsubxiphoid-videothoracoscopic "maximal" thymectomyoperative technique and early results. Ann Thorac Surg 2004;78:404-9.

11. Yoshino I, Hashizume M, Shimada M, et al. Thoracoscopic thymomectomy with the da Vinci computer-enhanced surgical system.J Thorac Cardiovasc Surg 2001;122:783-5.

12. Ashton RC, McGinnis KM, Connery CP, et al. Totally Endoscopic Robotic Thymectomy for Myasthenia Gravis. Ann Thorac Surg 2003;75:569-71.

13. O'Sullivan KE, Kreaden US, Hebert AE, et al. A systematic review of robotic versus open and video assisted

doi: 10.21037/jovs-20-128

Cite this article as: Montagne F, Delacour D, Fommarty M, Chaari Z, Papet E, Baste JM. Robotic radical thymothymectomy for thymomatous myasthenia gravis in the elderly: case report and mini-review. J Vis Surg 2021;7:20. thoracoscopic surgery (VATS) approaches for thymectomy. Ann Cardiothorac Surg 2019;8:174-93.

14. Kneuertz PJ, Kamel MK, Stiles BM, et al. Robotic Thymectomy Is Feasible for Large Thymomas: A Propensity-Matched Comparison. Ann Thorac Surg 2017;104:1673-8.

15. Jaretzki A, Barohn RJ, Ernstoff RM, et al. Myasthenia gravis: recommendations for clinical research standards. Task Force of the Medical Scientific Advisory Board of the Myasthenia Gravis Foundation of America. Neurology 2000;55:16-23.

16. Li F, Ismail M, Elsner A, et al. Surgical Techniques for Myasthenia Gravis: Robotic-Assisted Thoracoscopic Surgery. Thorac Surg Clin 2019;29:177-86.

17. Friedant AJ, Handorf EA, Su S, et al. Minimally Invasive versus Open Thymectomy for Thymic Malignancies: Systematic Review and Meta-Analysis. J Thorac Oncol 2016;11:30-8.

18. Marulli G, Maessen J, Melfi F, et al. Multi-institutional European experience of robotic thymectomy for thymoma. Ann Cardiothorac Surg 2016;5:18-25.

19. Buentzel J, Heinz J, Hinterthaner M, et al. Robotic versus thoracoscopic thymectomy: The current evidence. Int J Med Robot 2017;13:e1847.

20. Fok M, Bashir M, Harky A, et al. Video-Assisted Thoracoscopic Versus Robotic-Assisted Thoracoscopic Thymectomy Systematic Review and Meta-analysis. Innovations 2017;12:259-64. 